06

\title{
Нелинейная динамика параметрических осцилляций экситон-поляритонов в полупроводниковом микрорезонаторе при учете затухания
}

\author{
() О.Ф. Васильева, А.П. Зинган, В.В. Васильев \\ Приднестровский государственный университет им. Т.Г. Шевченко, \\ МД 3300 Тирасполь, Молдова \\ e-mail: florina_of@mail.ru
}

Поступила в редакцию 18.09.2019 г.

В окончательной редакции 14.10.2019 г.

Принята к публикации 28.10.2019 г.

Изучена динамика поляритонов в микрорезонаторе в режиме параметрического осциллятора, когда накачка осуществляется двумя лазерными импульсами с близкими частотами. Найдены аналитические решения системы нелинейных дифференциальных уравнений при равных константах затухания.

Ключевые слова: экситон-поляритоны, накачка, сигнальная и холостая моды.

DOI: $10.21883 /$ OS.2020.02.48969.269-19

\section{Введение}

Смешанные экситон-фотонные состояния в плоских полупроводниковых микрорезонаторах с квантовыми ямами в активном слое представляют собой новый класс квазидвумерных квазичастиц с уникальными свойствами [1-13]. Такие состояния называют микрорезонаторными экситон-поляритонами. Они возникают благодаря сильной связи экситонов с собственными модами электромагнитного излучения микрорезонатора. В условиях сильной связи экситонная и фотонная моды расталкиваются, и возникают верхняя и нижняя микрорезонаторные поляритонные моды. Непараболичность нижней поляритонной ветви допускает возникновение параметрического процесса, в результате которого два поляритона накачки рассеиваются в сигнальную и холостую моды с сохранением энергии и импульса. Поэтому огромный интерес вызывает поляритон-поляритонное рассеяние, благодаря которому экситон-поляритонная система демонстрирует сильные нелинейные свойства [6-13]. Такие нелинейности были обнаружены в спектрах люминесценции микрорезонаторов [14-18] при резонансном возбуждении нижней поляритонной ветви, которые объяснялись четырехволновым смешением или параметрическим рассеянием фотовозбужденных поляритонов накачки в сигнальную и холостую моды. Экспериментально идентифицированы два механизма нелинейности - это поляритонное параметрическое рассеяние $[6,19,20]$ и голубой сдвиг поляритонной дисперсии [2,5]. Используя pump-probe-метод, в [8,9] впервые наблюдалось параметрическое усиление в микрорезонаторе при возбуждении нижней поляритонной ветви пикосекундным импульсом накачки под углом падения $16.5^{\circ}$. После возбуждения (с небольшой задержкой) нижней поляритонной ветви дополнительно слабым пробным импульсом, падавшим нормально, обнаружилось, что этот импульс в отражении усиливался более чем в 70 раз. При этом появлялась также холостая мода под углом в $35^{\circ}$. Именно для этих углов выполнялись резонансные условия.

Результаты экспериментов [8,9] были воспроизведены также в [21] и моделировались в [7] с использованием механизма поляритон-поляритонного рассеяния. Аналогичные процессы наблюдались в [22] при использовании двух пучков накачки под углами в $\pm 45^{\circ}$ и пробного пучка под углом $0^{\circ}$. Режим параметрического осциллятора наблюдался в $[9,14]$ при непрерывном возбуждении нижней поляритонной ветви излучением накачки под „магическим“ углом в $16^{\circ}$ без пробного импульса. Выше пороговой интенсивности наблюдались сильные пучки сигнальной и холостой мод под углами $0^{\circ}$ и $35^{\circ}$ соответственно.

В [20] обнаружена сильная и необычная зависимость поляризации света, излучаемого микрорезонатором, от поляризации накачки. Эта зависимость интерпретируется с использованием псевдоспиновой модели в рамках квазиклассического формализма, где параметрическое рассеяние описывается как резонансное четырехволновое смешение. В [23-25] наблюдалось бистабильное пропускание излучения в зависимости от интенсивности накачки при возбуждении экситон-поляритонов в микрорезонаторе. Отметим, что процесс параметрического рассеяния наблюдался как при импульсном [20,26], так и при непрерывном [14,16,27] возбуждении.

Описание поляритонных параметрических осцилляторов и усилителей представлено в ряде работ $[2,5,7,8,12,13,28]$. Экситон-экситонные взаимодействия играют ключевую роль в сильных нелинейностях, присутствующих в поляритонных системах микрорезонатора. Первой попыткой контроля этих взаимодействий было использование концепции диполяритонов [29] пу- 
тем включения двойных асимметричных квантовых ям в электрически смещенный микрорезонатор. Как прямые, так и непрямые экситоны соединяются с одной и той же модой резонатора, образуя новый тип поляритона с аналогичными свойствами по отношению к экситон-поляритонной системе. В [30] теоретически исследовалась динамика диполяритонов при учете трех каналов рассеяния. Получены апериодические и периодические режимы эволюции диполяритонов накачки в диполяритоны сигнальной и холостой мод. В [31] путем объединения широких квантовых ям в простом волноводе наблюдалось образование диполярных поляритонов. Основным ограничением исследуемых поляритонных систем по сравнению с их атомными аналогами для изучения сильно коррелированных явлений и физики многих тел является их относительно слабое двухчастичное взаимодействие по сравнению с хаосом. В [32] показаны новые возможности усиления таких локальных взаимодействий и нелинейностей путем настройки экситон-поляритонного дипольного момента в электрически смещенных полупроводниковых микрорезонаторах, включающих широкие квантовые ямы.

Микрорезонаторные экситон-поляритоны обладают бистабильностью при низких оптических мощностях благодаря их сильным нелинейностям [24]. Поляризационная зависимость нелинейностей вызывает поляризационную мультистабильность [33,34], которая может быть использована для создания спиновых запоминающих устройств [35], логических вентилей [36,37] или переключателей [38].

В [39] теоретически предсказан и экспериментально [40] получен самозахват экситон-поляритонных конденсатов, который объясняется образованием нового поляроноподобного состояния. Захваченное состояние экситон-поляритонов стабилизируется за счет рассеяния экситонов в поляритонном конденсате. В [41] анализируется взаимодействие экситон-поляритонных конденсатов в полупроводниковом микрорезонаторе с акустическими фононами. Показано, что параметрическая неустойчивость в системе приводит к генерации когерентной акустической волны и дополнительных поляритонных гармоник.

В [42-46] при исследовании свойств оптического параметрического осциллятора использовались два одинаковых фотона накачки на нижней ветви поляритонного закона дисперсии. Однако в $[47,48]$ было показано, что два различных пучка накачки можно конвертировать в два вырожденных по частоте фотона сигнальной и холостой мод. Наличие двух различных пучков накачки дает большие возможности для генерации сигнального и холостого пучков с наперед заданными свойствами. В [49] теоретически изучена динамика поляритонов, когда накачка осуществляется двумя лазерами с близкими частотами без учета затухания в среде. Найдены апериодические и периодические режимы превращения пары поляритонов накачки в поляритоны сигнальной и холостой мод. Показано, что введение двух независимых

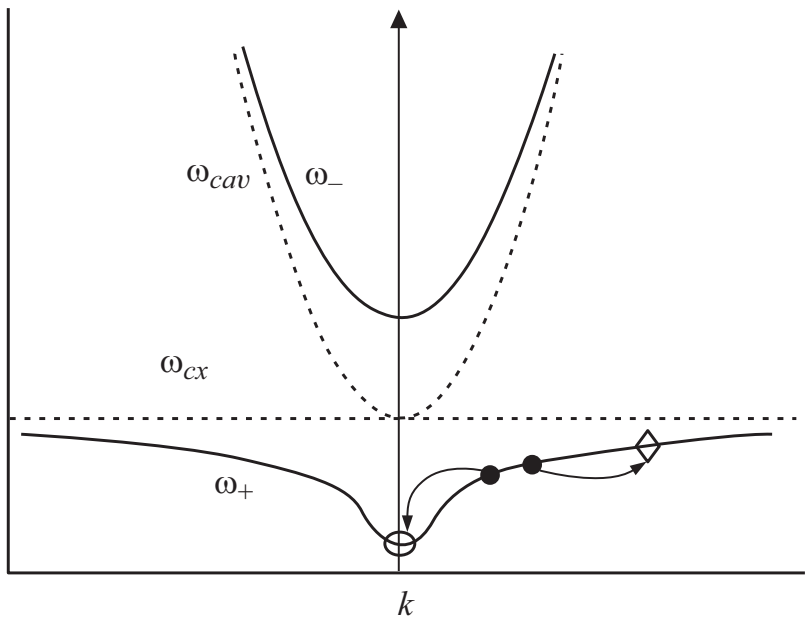

Рис. 1. Энергии поляритонов верхней и нижней ветвей $\left(\omega_{ \pm}\right)$. Дисперсия собственных частот микрорезонатора $\omega_{c a v}$ и экситона $\omega_{e x}$. Два поляритона накачки рассеиваются в сигнальную и холостую моды.

накачек приводит к увеличению степеней свободы системы.

\section{Постановка задачи. Основные уравнения}

Цель данной работы - изучить изменение во времени плотности поляритонов при накачке нижней ветви в двух близких по энергии точках закона дисперсии при учете затухания. Мы будем считать, что оба пучка накачки различаются по амплитуде (интенсивности), однако энергии фотонов различаются слабо. Предполагается, что поляритоны возбуждаются на нижней ветви закона дисперсии под „магическим углом“ (рис. 1). В [4,5] показано, что процесс параметрического рассеяния двух поляритонов накачки в сигнальную и холостую моды описывается гамильтонианом вида

$$
\begin{aligned}
\frac{1}{\hbar} H & =\omega_{p_{1}} \hat{a}_{p_{1}}^{+} \hat{a}_{p_{1}}+\omega_{p_{2}} \hat{a}_{p_{2}}^{+} \hat{a}_{p_{2}}+\omega_{s} \hat{a}_{s}^{+} \hat{a}_{s}+\omega_{i} \hat{a}_{i}^{+} \hat{a}_{i} \\
& +\mu\left(\hat{a}_{s}^{+} \hat{a}_{i}^{+} \hat{a}_{p_{1}} \hat{a}_{p_{2}}+\hat{a}_{p_{1}}^{+} \hat{a}_{p_{2}}^{+} \hat{a}_{s} \hat{a}_{i}\right),
\end{aligned}
$$

где $\omega_{p_{1}}, \omega_{p_{2}}, \omega_{s}$ и $\omega_{i}-$ собственные частоты поляритонов накачки первого и второго импульсов, сигнальной и холостой мод соответственно, $\hat{a}_{p_{1}}, \hat{a}_{p_{2}}, \hat{a}_{s}, \hat{a}_{i}-$ операторы уничтожения поляритонов, $\mu$ - константа параметрической поляритон-поляритонной конверсии. Используя (1), легко получить систему гайзенберговских уравнений для операторов $\hat{a}_{p_{1}}, \hat{a}_{p_{2}}, \hat{a}_{s}, \hat{a}_{i}$. Усредняя эту систему уравнений и используя приближение среднего поля (mean field approximation), применимость которого обоснована в [50], для комплексных амплитуд поляритонов $a_{p_{1}}=\left\langle\hat{a}_{p_{1}}\right\rangle, a_{p_{2}}=\left\langle\hat{a}_{p_{2}}\right\rangle, a_{s}=\left\langle\hat{a}_{s}\right\rangle$ и $a_{i}=\left\langle\hat{a}_{i}\right\rangle$ можно получить следующую систему нелинейных эволюцион- 
ных уравнений:

$$
\begin{aligned}
i \dot{a}_{p_{1}} & =\left(\omega_{p_{1}}-i \gamma_{p_{1}}\right) a_{p_{1}}+\mu a_{p_{2}}^{*} a_{s} a_{i}, \\
i \dot{a}_{p_{2}} & =\left(\omega_{p_{2}}-i \gamma_{p_{2}}\right) a_{p_{2}}+\mu a_{p_{1}}^{*} a_{s} a_{i}, \\
i \dot{a}_{s} & =\left(\omega_{s}-i \gamma_{s}\right) a_{s}+\mu a_{p_{1}} a_{p_{2}} a_{i}^{*}, \\
i \dot{a}_{i} & =\left(\omega_{i}-i \gamma_{i}\right) a_{i}+\mu a_{p_{1}} a_{p_{2}} a_{s}^{*},
\end{aligned}
$$

где $\gamma_{p_{1}}, \gamma_{p_{2}}, \gamma_{s}$ и $\gamma_{i}$ - константы затухания соответствующих поляритонных состояний, которые мы вводим феноменологически. Систему уравнений (2) дополним начальными условиями, которые запишем в виде

$$
\begin{aligned}
a_{p_{1} \mid t=0} & =a_{p_{1} 0} \exp \left(i \varphi_{p_{1} 0}\right), \\
a_{p_{2} \mid t=0} & =a_{p_{2} 0} \exp \left(i \varphi_{p_{2} 0}\right), \\
a_{s \mid t=0} & =a_{s 0} \exp \left(i \varphi_{s 0}\right), \\
a_{i \mid t=0} & =a_{i 0} \exp \left(i \varphi_{i 0}\right),
\end{aligned}
$$

где $a_{p_{1} 0}, a_{p_{2} 0}, a_{s 0}, a_{i 0}$ и $\varphi_{p_{1} 0}, \varphi_{p_{2} 0}, \varphi_{s 0}, \varphi_{i 0}$ - действительные амплитуды и фазы поляритонов в начальный момент времени.

Введем далее в рассмотрение плотности поляритонов $n_{p_{1}}=a_{p_{1}}^{*} a_{p_{1}}, n_{p_{2}}=a_{p_{2}}^{*} a_{p_{2}}, n_{s}=a_{s}^{*} a_{s}, n_{i}=a_{i}^{*} a_{i}$ и две компоненты „поляризации“ $Q=i\left(a_{p_{1}} a_{p_{2}} a_{s}^{*} a_{i}^{*}-\right.$ $\left.-a_{s} a_{i} a_{p_{1}}^{*} a_{p_{2}}^{*}\right)$ и $R=a_{p_{1}} a_{p_{2}} a_{s}^{*} a_{i}^{*}+a_{s} a_{i} a_{p_{1}}^{*} a_{p_{2}}^{*}$.

Используя (2), для введенных функций получаем следуюшую систему нелинейных дифференциальных уравнений:

$$
\begin{gathered}
\dot{n}_{p_{1}}=-2 \gamma_{p_{1}} n_{p_{1}}+\mu Q, \dot{n}_{p_{2}}=-2 \gamma_{p_{2}} n_{p_{2}}+\mu Q, \\
\dot{n}_{s}=-2 \gamma_{s} n_{s}-\mu Q, \dot{n}_{i}=-2 \gamma_{i} n_{i}-\mu Q, \\
\dot{Q}=\Delta R+2 \mu\left(n_{s} n_{i}\left(n_{p_{2}}+n_{p_{1}}\right)-n_{p_{1}} n_{p_{2}}\left(n_{i}+n_{s}\right)\right)-\Gamma Q, \\
\dot{R}=-\Delta Q-\Gamma R,
\end{gathered}
$$

где $\Delta=\omega_{p_{1}}+\omega_{p_{2}}-\omega_{s}-\omega_{i}-$ расстройка резонанса, $\Gamma=\gamma_{p_{1}}+\gamma_{p_{2}}+\gamma_{s}+\gamma_{i}$. Используя (3), начальные условия для этих функций можно записать в виде

$$
\begin{gathered}
n_{p_{1} \mid t=0} \equiv n_{p_{1} 0}=\left|a_{p_{1} 0}\right|^{2}, n_{p_{2} \mid t=0} \equiv n_{p_{2} 0}=\left|a_{p_{2} 0}\right|^{2}, \\
n_{s \mid t=0} \equiv n_{s 0}=\left|a_{s 0}\right|^{2}, n_{i \mid t=0} \equiv n_{i 0}=\left|a_{i 0}\right|^{2}, \\
Q_{\mid t=0} \equiv Q_{0}=2 \sqrt{n_{p_{1} 0} n_{p_{2} 0} n_{s 0} n_{i 0}} \sin \theta_{0}, \\
R_{\mid t=0} \equiv R_{0}=2 \sqrt{n_{p_{1} 0} n_{p_{2} 0} n_{s 0} n_{i 0}} \cos \theta_{0},
\end{gathered}
$$

где $\theta_{0}=\varphi_{s 0}+\varphi_{i 0}-\varphi_{p_{1} 0}-\varphi_{p_{2} 0}-$ начальная разность фаз.

Получить точные аналитические решения системы уравнений (4) в общем виде не удается. Однако, если рассмотреть случай точного резонанса $(\Delta=0)$ и считать равными константы затухания $\left(\gamma_{p_{1}}=\gamma_{p_{2}}=\gamma_{s}=\right.$ $\left.=\gamma_{i} \equiv \gamma\right)$, такие решения можно получить. Для этого необходимо ввести новые функции: $n_{p_{1}}=N_{p_{1}} \exp (-2 \gamma t)$,
$n_{p_{2}}=N_{p_{2}} \exp (-2 \gamma t), n_{s}=N_{s} \exp (-2 \gamma t), n_{i}=N_{i} \exp (-2 \gamma t)$, $Q=q \exp (-4 \gamma t), R=r \exp (-4 \gamma t)$ и новую переменную

$$
\xi=\frac{1-\exp (-2 \gamma t)}{2 \gamma} \text {. }
$$

Тогда система уравнений (4) приводится к виду

$$
\begin{gathered}
\frac{d N_{p_{1}}}{d \xi}=\frac{d N_{p_{2}}}{d \xi}=\mu q, \\
\frac{d N_{s}}{d \xi}=\frac{d N_{i}}{d \xi}=-\mu q, \\
\frac{d q}{d \xi}=2 \mu\left(N_{s} N_{i}\left(N_{p_{2}}+N_{p_{1}}\right)-N_{p_{1}} N_{p_{2}}\left(N_{i}+N_{s}\right)\right), \frac{d r}{d \xi}=0 .
\end{gathered}
$$

Если принять переменную $\xi$ за время, то систему уравнений (6) можно рассматривать как „консервативную“, решения которой легко найти.

Из (6) нетрудно получить следующие интегралы движения:

$$
\begin{gathered}
N_{p_{1}}-N_{p_{2}}=N_{p_{1} 0}-N_{p_{2} 0}, N_{s}-N_{i}=N_{s 0}-N_{i 0}, \\
N_{p_{1}}+N_{s}=N_{p_{1} 0}+N_{s 0}, N_{p_{2}}+N_{i}=N_{p_{2} 0}+N_{i 0}, \\
q^{2}+r^{2}=4 N_{p_{1}} N_{p_{2}} N_{i} N_{s}, \quad r=r_{0} .
\end{gathered}
$$

Тогда систему уравнений (4) можно привести к одному нелинейному дифференциальному уравнению для плотности поляритонов накачки первого импульса $N_{p_{1}}$ :

$$
\frac{1}{2}\left(\frac{d N_{p_{1}}}{d \xi}\right)^{2}+W\left(N_{p_{1}}\right)=E_{0}
$$

где

$$
\begin{gathered}
W\left(N_{p_{1}}\right)=-2 N_{p_{1}}\left(N_{p_{1}}-n_{p_{1} 0}+n_{p_{2} 0}\right)\left(n_{p_{1} 0}+n_{s 0}-N_{p_{1}}\right) \\
\times\left(n_{p_{1} 0}+n_{i 0}-N_{p_{1}}\right) \\
\quad E_{0}=-n_{p_{1}} n_{p_{2} 0} n_{s 0} n_{i 0} \cos ^{2} \theta_{0}
\end{gathered}
$$

Здесь уравнение (8) представляет собой закон сохранения энергии для нелинейного осциллятора, где слагаемые слева играют роль кинетической и потенциальной энергий соответственно, а $E_{0}$ играет роль полной энергии осциллятора. Колебания осциллятора возможны в той области значений $N_{p_{1}}$, в которой $W\left(N_{p_{1}}\right) \leq E_{0}$. Качественно поведение функции $N_{p_{1}}(\xi)$ можно установить, изучая поведение потенциальной энергии $W$ от $N_{p_{1}}$ при различных значениях параметров. Вид решения существенно определяется корнями алгебраического уравнения четвертой степени $W\left(N_{p_{1}}\right)=E_{0}$, которые зависят от значений параметров $n_{p_{1} 0}, n_{p_{2} 0}, n_{s 0}, n_{i 0}$ и $\theta_{0}$.

Рассмотрим сначала случай, когда начальная разность фаз равна $\pi / 2$. Пусть начальная плотность поляритонов накачки второго импульса больше, чем начальная плотность поляритонов накачки первого импульca $\left(n_{p 0_{2}}>n_{p 0_{1}}\right)$, и начальная плотность поляритонов сигнальной моды больше, чем начальная плотность 
поляритонов холостой моды $\left(n_{s}>n_{i 0}\right)$. Тогда решение уравнения (8) запишется в виде

$$
n_{p_{1}}=n_{p_{1} 0} \exp (-2 \gamma t) \frac{\left(n_{p 0_{1}}-n_{p 0_{2}}\right)\left(n_{p 0_{1}}+n_{i 0}\right)}{n_{p 0_{2}}-n_{p 0_{1}} \operatorname{sn}^{2}\left( \pm s+F\left(\varphi_{0}, k\right)\right)}
$$

где $\operatorname{sn}(x)$ - эллиптическая функция Якоби с модулем $k$ [51,52], а величины $k, \varphi_{0}$ и $s$ выражаются формулами

$$
\begin{gathered}
k^{2}=\frac{\left(n_{p 0_{2}}+n_{s 0}\right)\left(n_{p 0_{1}}+n_{i 0}\right)}{\left(n_{p 0_{1}}+n_{s 0}\right)\left(n_{p 0_{2}}+n_{i 0}\right)}, \\
\varphi_{0}=\arcsin \sqrt{\frac{\left(n_{p 0_{2}}+n_{i 0}\right) n_{p 0_{1}}}{\left(n_{p 0_{1}}+n_{i 0}\right) n_{p 0_{2}}}} \\
s=\sqrt{\left(n_{p 0_{1}}+n_{s 0}\right)\left(n_{p 0_{2}}+n_{i 0}\right)} \mu \frac{1-\exp (-2 \gamma t)}{2 \gamma} .
\end{gathered}
$$

Теперь рассмотрим эволюцию системы в случае, когда в начальный момент времени начальная плотность поляритонов накачки первого импульса больше, чем начальная плотность поляритонов накачки второго импульса $\left(n_{p 0_{1}}>n_{p 0_{2}}\right)$, и, определенности ради, $n_{s 0}<n_{i 0}$. Тогда решение уравнения (8) получаем в виде

$$
\begin{aligned}
n_{p_{1}} & =n_{p_{1} 0} \exp (-2 \gamma t) \\
& \times \frac{\left(n_{p 0_{1}}-n_{p 0_{2}}\right)\left(n_{p 0_{1}}+n_{i 0}\right)}{n_{p_{1} 0}-n_{p_{2} 0}-\left(n_{p 0_{2}}+n_{i 0}\right) \operatorname{sn}^{2}\left( \pm d+F\left(\varphi_{0}, k\right)\right)},
\end{aligned}
$$

где величины $k, \varphi_{0}$ и $d$ выражаются формулами

$$
\begin{gathered}
k^{2}=\frac{\left(n_{p 0_{1}}+n_{s 0}\right)\left(n_{p 0_{2}}+n_{i 0}\right)}{\left(n_{p 0_{2}}+n_{s 0}\right)\left(n_{p 0_{1}}+n_{i 0}\right)}, \\
\varphi=\arcsin \sqrt{\frac{\left(n_{p 0_{1}}+n_{i 0}\right) n_{p 0_{2}}}{\left(n_{p 0_{2}}+n_{i 0}\right) n_{p 0_{1}}}} \\
d=\sqrt{\left(n_{p 0_{2}}+n_{s 0}\right)\left(n_{p 0_{1}}+n_{i 0}\right)} \mu \frac{1-\exp (-2 \gamma t)}{2 \gamma} .
\end{gathered}
$$

Из рис. 2, $a, b$ видно, что плотность поляритонов накачки первого импульса в обоих случаях убывает со временем, осциллируя. Огибающие максимумов и минимумов осцилляций экспоненциально убывают со временем. При этом расстояние между двумя соседними пиками (либо минимумами) растет со временем. При больших $\gamma$ падение плотности поляритонов характеризуется ограниченным сверху числом осцилляций на начальном этапе эволюции, после чего устанавливается экспоненциальный спад плотности без осцилляций. Это обусловлено тем, что аргумент эллиптического синуса на больших временах стремится к постоянной величине, значение эллиптического синуса перестает осциллировать во времени, и временная эволюция плотности поляритонов при этом определяется только экспоненциальным множителем $\exp (-2 \gamma t)$.
Наконец, если начальная плотность поляритонов накачки первого импульса равна начальной плотности поляритонов накачки второго импульса $\left(n_{p 0_{1}}=n_{p 0_{2}}\right)$, то решение уравнения (8) получается в виде

$$
\begin{aligned}
& n_{p_{1}}=n_{p_{1} 0} \exp (-2 \gamma t) \\
& \quad \frac{4 a b}{C \exp ( \pm z)+2(a+b)+\frac{(a-b)^{2}}{C} \exp (\mp z)},
\end{aligned}
$$

где

$$
\begin{gathered}
a=n_{p 0_{1}}+n_{s 0}, \quad b=n_{p 0_{1}}+n_{i 0}, \\
C=\frac{\sqrt{n_{i 0}\left(n_{p 0_{1}}+n_{s 0}\right)+n_{s 0}\left(n_{p 0_{1}}+n_{i 0}\right)}}{n_{p 0_{1}}}, \\
z=2 \mu \sqrt{a b} \frac{1-\exp (-2 \gamma t)}{2 \gamma},
\end{gathered}
$$

которое при равенстве начальных плотностей поляритонов сигнальной и холостой мод $\left(n_{s 0}=n_{i 0}\right)$ дает

$$
\begin{aligned}
& n_{p_{1}}= \\
& \frac{n_{p 0_{1}}\left(n_{p 0_{1}}+n_{s 0}\right) \exp \left( \pm 2 \mu\left(n_{p 0_{1}}+n_{s 0}\right) \frac{1-\exp (-2 \gamma t)}{2 \gamma}\right)}{n_{s 0}+n_{p 0_{1}} \exp \left( \pm 2 \mu\left(n_{p 0_{1}}+n_{s 0}\right) \frac{1-\exp (-2 \gamma t)}{2 \gamma}\right)} \\
& \times \exp (-2 \gamma t) .
\end{aligned}
$$

Из (14) и рис. 2, с видно, что решение со знаком (+) монотонно убывает со временем, тогда как решение со знаком (-) сначала растет, в некоторый момент времени достигает своего максимума, затем монотонно убывает. На больших временах оба решения ведут себя одинаково. Решения со знаком $(+)$ и $(-)$ отличаются друг от друга только постоянной фазой, которая определяется начальными плотностями частиц и обусловлена двумя равными по величине, но противоположными по направлению начальными скоростями изменения функции $n_{p_{1}}(t)$. Качественно различное поведение этих решений на небольших временах обусловлено тем, что в первом случае плотность поляритонов накачки первого импульса убывает как из-за затухания, так и из-за апериодической конверсии поляритонов накачки в поляритоны сигнальной и холостой мод, тогда как во втором случае на начальном этапе имеет место сначала рост плотности поляритонов накачки первого импульса за счет превращения пар поляритонов сигнальной и холостой мод в поляритоны накачки. При этом такой рост на начальном этапе является более крутым, чем экспоненциальное убывание. Затем по мере истощения плотностей поляритонов сигнальной и холостой мод остается в сущности только экспоненциальное убывание из-за затухания. В общем, эволюция плотности поляритонов в конечном счете сводится к полному исчезновению всех поляритонов микрорезонатора (рис. 2,c). 

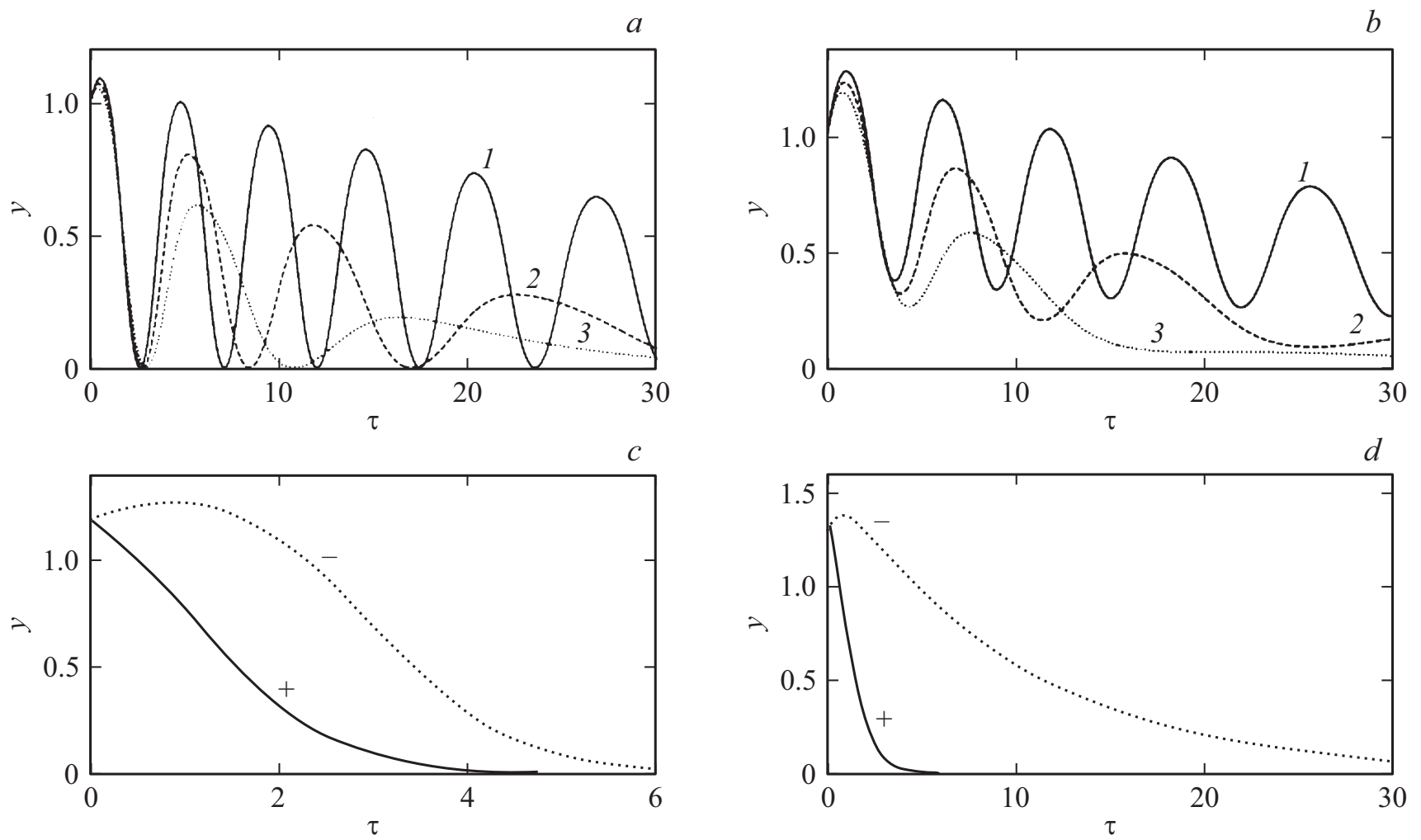

Рис. 2. Временная эволюция плотности поляритонов накачки первого импульса $y=\frac{n_{p_{1}}}{n_{p_{1} 0}}$ при начальной разности фаз, равной $\theta_{0}=\pi / 2$, а также при фиксированных начальных плотностях поляритонов накачки второго импульса, начальной плотности поляритонов сигнальной и холостой мод и коэффициентов затухания: (a) $n_{p_{2} 0} / n_{p_{1} 0}=1.5, n_{s 0} / n_{p_{1} 0}=0.3, n_{i 0} / n_{p_{1} 0}=0.1$, $\gamma=0.01$ (1), 0.03 (2), 0.05 (3); (b) $n_{p_{2} 0} / n_{p_{1} 0}=0.6, n_{s 0} / n_{p_{1} 0}=0.3, n_{i 0} / n_{p_{1} 0}=0.5, \gamma=0.01(1), 0.03(2), 0.05(3) ;(c) n_{p_{2} 0} / n_{p_{1} 0}=1$, $n_{s 0} / n_{p_{1} 0}=0.6, n_{i 0} / n_{p_{1} 0}=0.3, \gamma=0.01 ;(d) n_{p_{2} 0} / n_{p_{1} 0}=1, n_{s 0} / n_{p_{1} 0}=0.6, n_{i 0} / n_{p_{1} 0}=0.6, \gamma=0.01$. Здесь $\tau=\mu t / n_{p_{1} 0}$.

Из (15) и рис. 2, $d$ следует, что при $t \gg \gamma^{-1}$ экспоненциальный множитель $e^{-2 \gamma t}$ у плотностей частиц приводит к тому, что система поляритонов затухает со временем, и плотности всех частиц обращаются в нуль. Видно также, что предельные значения плотностей поляритонов при $t \rightarrow \infty$ теперь оказываются невозможными (решение со знаком $(-)$ ).

Рассмотрим теперь эволюцию системы при начальной разности фаз $\theta_{0}=0$. Если начальная плотность одного из поляритонов накачки, например второго импульса, меньше либо равна начальной плотности поляритонов холостой моды $\left(n_{p 0_{2}} \leq n_{i 0}\right)$, при определенном соотношении между начальными параметрами системы решение уравнения (8) имеет вид $n_{p_{1}}=n_{p_{1} 0} \exp (-2 \gamma t)$, т. е. совпадает с начальным условием, что обусловлено пересечением двух средних корней уравнения $W\left(N_{p_{1}}\right)=E_{0}$. Поэтому решение уравнения (8) не будет содержать фазового сдвига. В отсутствие вырождения двух средних корней уравнения $W\left(N_{p_{1}}\right)=E_{0}$ возникают два случая эволюции. В зависимости от соотношения между параметрами $n_{p_{1} 0}$, $n_{p_{2} 0}, n_{i 0}, n_{s} 0$ в первом случае корни упорядочиваются так, что $y_{1}>n_{p_{1} 0}>y_{m}>y_{4}$, а во втором $y_{1}>y_{M}>n_{p_{1} 0}>y_{4}$.
Решение уравнения (8) в случае, когда $y_{1}>n_{p_{1} 0}>$ $>y_{m}>y_{4}$, имеет вид

$$
\begin{aligned}
& n_{p_{1}}=n_{p_{1} 0} \exp (-2 \gamma t) \times \\
& \times \frac{1-\frac{y_{1}\left(\dot{n}_{p_{1}}-y_{m}\right)}{y_{1}-y_{m}} \operatorname{sn}^{2}\left(\mu \sqrt{\left(y_{1}-y_{m}\right)\left(n_{p_{1} 0}-y_{4}\right)} \frac{1-\exp (-2 \gamma t)}{2 \gamma}\right)}{1-\frac{n_{p_{1} 0}-y_{m}}{y_{1}-y_{m}} \operatorname{sn}^{2}\left(\mu \sqrt{\left(y_{1}-y_{m}\right)\left(n_{p_{1} 0}-y_{4}\right)} \frac{1-\exp (-2 \gamma t)}{2 \gamma}\right)},
\end{aligned}
$$

где

$$
k^{2}=\frac{\left(y_{1}-y_{4}\right)\left(n_{p_{1} 0}-y_{m}\right)}{\left(y_{1}-y_{m}\right)\left(n_{p_{1} 0}-y_{4}\right)} .
$$

Во втором случае, когда $y_{1}>y_{M}>n_{p_{1} 0}>y_{4}$, соответственно получаем

$$
\begin{aligned}
& n_{p_{1}}=n_{p_{1} 0} \exp (-2 \gamma t) \times \\
& \times \frac{1-\frac{y_{4}\left(y_{M}-n_{p_{1}}\right)}{y_{M}-y_{4}} \operatorname{sn}^{2}\left(\mu \sqrt{\left(y_{1}-n_{p_{1} 0}\right)\left(y_{M}-y_{4}\right)} \frac{1-\exp (-2 \gamma t)}{2 \gamma}\right)}{1-\frac{y_{M}-n_{p_{1} 0} 0}{y_{M}-y_{4}} \operatorname{sn}^{2}\left(\mu \sqrt{\left(y_{1}-n_{p_{1} 0}\right)\left(y_{M}-y_{4}\right)} \frac{1-\exp (-2 \gamma t)}{2 \gamma}\right)},
\end{aligned}
$$

где

$$
k^{2}=\frac{\left(y_{1}-y_{4}\right)\left(y_{M}-n_{p_{1} 0}\right)}{\left(y_{1}-n_{p_{1} 0}\right)\left(y_{M}-y_{4}\right)} .
$$




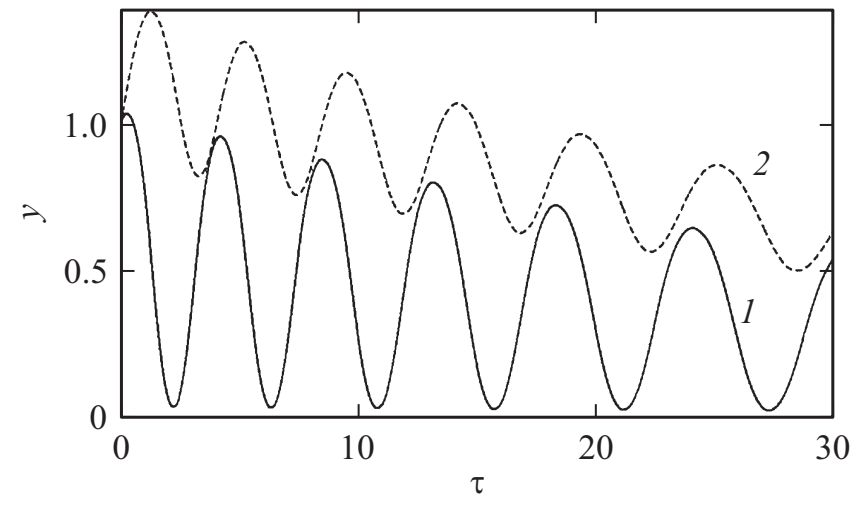

Рис. 3. Временная эволюция плотности поляритонов накачки первого импульса $y=\frac{n_{p_{1}}}{n_{p_{1} 0}}$ при начальной разности фаз, равной $\theta_{0}=0$, а также при фиксированных начальных плотностях поляритонов накачки второго импульса, начальной плотности поляритонов сигнальной и холостой мод и коэффициентов затухания: $n_{p_{2} 0} / n_{p_{1} 0}=1.5, n_{s 0} / n_{p_{1} 0}=0.3, n_{i 0} / n_{p_{1} 0}=0.1$, $\gamma=0.01(1) ; n_{p_{2}} / n_{p_{1} 0}=0.2, \quad n_{s 0} / n_{p_{1} 0}=0.6, \quad n_{i 0} / n_{p_{1} 0}=0.7$, $\gamma=0.01$ (2). Здесь $\tau=\mu t / n_{p_{1} 0}$.

Из рис. 3 видно, что со временем амплитуда колебаний этих решений экспоненциально затухает, и при $t \gg \gamma^{-1}$ нормированная плотность поляритонов накачки первого импульса обращается в нуль.

Таким образом, из представленных результатов следует, что временная эволюция плотности поляритонов накачки при начальной разности фаз $\theta_{0}=\pi / 2$ отличается от временной эволюции при $\theta_{0}=0$. Поэтому представляет интерес исследование особенностей временной эволюции при произвольном значении начальной разности фаз $\theta_{0}$. Уравнение $W\left(N_{p_{1}}\right)=E_{0}$ имеет четыре действительных корня, которые расположим в порядке $y_{1}>y_{M}>y_{m}>y_{4}$. Значения этих корней определяются параметрами $n_{p_{1} 0}, n_{p_{2} 0}, n_{s 0}, n_{i 0}, \theta_{0}$. В этом случае решение имеет вид

$$
\begin{aligned}
& n_{p_{1}}=n_{p_{1} 0} \exp (-2 \gamma t) \times \\
& \times \frac{y_{M}-\frac{y_{1}\left(y_{M}-y_{m}\right)}{y_{1}-y_{m}} \operatorname{sn}^{2}\left(\mu \nu \frac{1-\exp (-2 \gamma t)}{2 \gamma} \pm f\left(\varphi_{0}, k\right)\right)}{1-\frac{y_{M}-y_{m}}{y_{1}-y_{m}} \operatorname{sn}^{2}\left(\mu \nu \frac{1-\exp (-2 \gamma t)}{2 \gamma} \pm f\left(\varphi_{0}, k\right)\right)},
\end{aligned}
$$

где $f\left(\varphi_{0}, k\right)=F\left(\varphi_{0}, k\right)-K(k), F\left(\varphi_{0}, k\right)$ - неполный эллиптический интеграл первого рода с модулем $k$ и параметром $\varphi_{0}, K(k)$ - полный эллиптический интеграл $[48,49]$. Величины $k \varphi_{0}$ и $v$ выражаются соотношениями

$$
\begin{gathered}
k^{2}=\frac{\left(y_{1}-y_{4}\right)\left(y_{M}-y_{m}\right)}{\left(y_{1}-y_{m}\right)\left(y_{M}-y_{4}\right)}, \\
\varphi_{0}=\arcsin \sqrt{\frac{\left(y_{M}-y_{4}\right)\left(n_{p_{1} 0}-y_{m}\right)}{\left(y_{M}-y_{m}\right)\left(n_{p_{1} 0}-y_{4}\right)}}, \\
v=\sqrt{\left(y_{1}-y_{m}\right)\left(y_{M}-y_{4}\right)} .
\end{gathered}
$$

Из (20), (21) и рис. 4 видно, что плотность поляритонов накачки первого импульса $n_{p_{1}}(t)$, осциллируя, монотонно убывает со временем. Расстояние между двумя ближайшими точками на рис. 4, колеблющимися в одной и той же фазе, монотонно растет со временем. При начальной разности фаз $\theta_{0}$, стремящейся к величине $(2 k+1) \pi / 2(k=0,1,2, \ldots)$, временная эволюция становится апериодической, и осцилляции плотности поляритонов отсутствуют (рис. $4, b, c)$.

Отметим, что плотности поляритонов сигнальной, холостой мод и поляритонов накачки второго импульса точно так же, осциллируя, убывают со временем и обращаются в нуль на больших временах $\left(t \gg \gamma^{-1}\right)$.

При произвольных значениях констант затухания $\gamma_{p_{1}}$, $\gamma_{p_{2}}, \gamma_{s}$ и $\gamma_{i}$ решения уравнений (4) можно получить только численными методами. На рис. 5 представлены графики временной эволюции плотности поляритонов накачки первого импульса при произвольных значениях
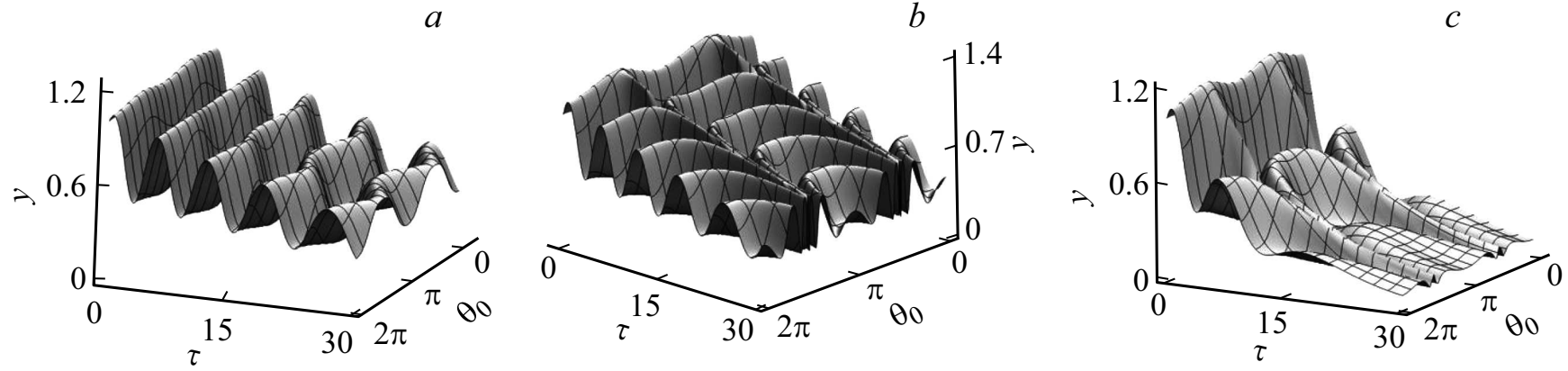

Рис. 4. Временная эволюция плотности поляритонов накачки первого импульса $y=\frac{n_{p_{1}}}{n_{p_{1} 0}}$ в зависимости от начальной разности фаз $\theta_{0}$ при фиксированных начальных плотностях поляритонов накачки второго импульса, поляритонов сигнальной и холостой мод и коэффициентов затухания: $(a) n_{p_{2} 0} / n_{p_{1} 0}=0.5, n_{s 0} / n_{p_{1} 0}=0.3, n_{i 0} / n_{p_{1} 0}=0.1, \gamma=0.01 ;(b) n_{p_{2} 0} / n_{p_{1} 0}=0.5, n_{s 0} / n_{p_{1} 0}=0.3$, $n_{i 0} / n_{p_{1} 0}=0.3, \gamma=0.01 ;$ (c) $n_{p_{2} 0} / n_{p_{1} 0}=0.5, n_{s 0} / n_{p_{1} 0}=0.3, n_{i 0} / n_{p_{1} 0}=0.3, \gamma=0.04$. Здесь $\tau=\mu t / n_{p_{1} 0}$. 


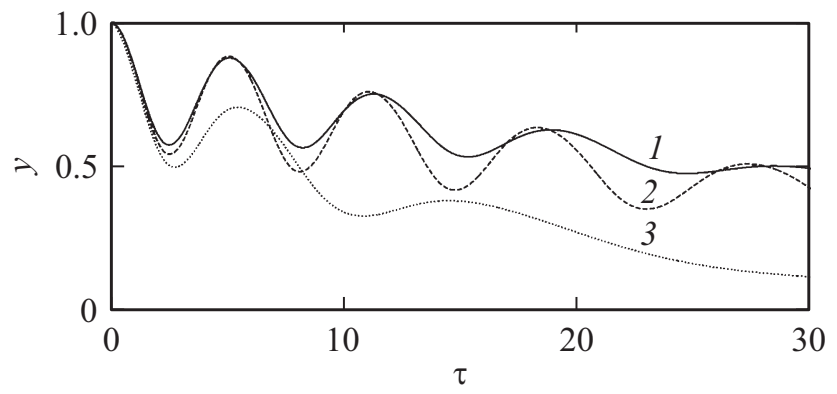

Рис. 5. Временная эволюция плотности поляритонов накачки первого импульса $y=\frac{n_{p_{1}}}{n_{p_{1} 0}}$ при начальной разности фаз, равной $\theta_{0}=0$, а также при фиксированных начальных плотностях поляритонов накачки второго импульса, начальной плотности поляритонов сигнальной и холостой мод $n_{p_{2} 0} / n_{p_{1} 0}=1.5$, $n_{s 0} / n_{p_{1} 0}=0.3, n_{i 0} / n_{p_{1} 0}=0.1$ и различных коэффициентов затухания соответствующих поляритонных состояний $\gamma_{p_{1}}=0.01$, $\gamma_{p_{2}}=0.04, \quad \gamma_{s}=0.02, \gamma_{i}=0.03(1) ; \gamma_{p_{1}}=0.01, \gamma_{p_{2}}=0.01$, $\gamma_{s}=0.02, \gamma_{i}=0.02(2) ; \gamma_{p_{1}}=0.03, \quad \gamma_{p_{2}}=0.04, \quad \gamma_{s}=0.05$, $\gamma_{i}=0.03$ (3). Здесь $\tau=\mu t / n_{p_{1} 0}$.

постоянных затухания $\gamma_{p_{1}}, \gamma_{p_{2}}, \gamma_{s}$ и $\gamma_{i}$. Видно, что в этом случае огибающие плотности поляритонов монотонно убывают со временем, причем скорость убывания существенно определяется соотношениями между константами затухания. Таким образом, качественно поведение системы не изменится.

\section{Заключение}

Таким образом, при накачке нижней поляритонной ветви в двух близких точках закона дисперсии при учете затухания в зависимости от начальной разности фаз, начальной плотности поляритонов накачки обоих импульсов, начальной плотности поляритонов сигнальной и холостой мод возможны различные режимы эволюции системы поляритонов: осцилляционное и экспоненциальное затухание амплитуды колебаний соответствующих поляритонных состояний. Учет затухания приводит к исчезновению поляритонов в системе.

\section{Конфликт интересов}

Авторы заявляют, что у них нет конфликта интересов.

\section{Список литературы}

[1] Kavokin A.V., Malpuech G. Thin Films and Nanostructures. Cavity Polaritons. / Ed. by Agranovich V.M., Taylor D. Amsterdam, 2003.

[2] Deng H., Haug H., Yamamoto Y. // Rev. Mod. Phys. 2010. V. 82. P. 1489.

[3] Kavokin A. // Appl. Phys. A. 2007. V. 89. P. 241.

[4] Glazov M.M., Kavokin K.V. // Phys. Rev. B. 2006. V. 73. P. 245317.
[5] Shelykh I.A., Johne R., Solnyshkov D.D., Kavokin A.V., Gippius N.A., Malpuech G. // Phys. Rev. B. 2007. V. 76. P. 155308.

[6] Whittaker D.M. // Phys. Rev. B. 2001. V. 63. P. 193305.

[7] Ciuti C., Schwendimann P., Deveaud B., Quattropani A. // Phys. Rev. B. 2000. V. 62. P. R4825.

[8] Savvidis P.G., Baumberg J.J., Stevenson R.M., Skolnick M.S., Whittaker D.M., Roberts J.S. // Phys. Rev. Lett. 2000. V. 84. P. 1547.

[9] Baumberg J.J., Savvidis P.G., Stevenson R.M., Tartakovskii A.I., Skolnick M.S., Whittaker D.M., Roberts J.S. // Phys. Rev. B. 2000. V. 62. P. R16247.

[10] Ciuti C. // Phys. Rev. B. 2004. V. 69. P. 245304.

[11] Schwendimann P., Ciuti C., Quattropani A. // Phys. Rev. B. 2003. V. 68. P. 165324.

[12] Savvidis P.G., Baumberg J.J., Porras D., Whittaker D.M., Skolnick M.S., Roberts J.S. // Phys. Rev. B. 2002. V. 65. P. 073309.

[13] Shelykh I.A., Kavokin A.V., Malpuech G. // Phys. Stat. Sol. B. 2005. V. 242. P. 2271.

[14] Stevenson R.M., Astratov V.N., Skolnick M.S., Whittaker D.M., Emam-Ismail M., Tartakovskii A.I., Savvidis P.G., Baumberg J.J., Roberts J.S. // Phys. Rev. Lett. 2000. V. 85. P. 3680 .

[15] Tartakovskii A.I., Krizhanovskii D.N., Malpuech G., EmamIsmail M., Chernenko A.V., Kavokin A.V., Kulakovskii V.D., Skolnick M.S., Roberts J.S. // Phys. Rev. B. 2003. V. 67. P. 165302.

[16] Tartakovskii A.I., Krizhanovskii D.N., Kulakovskii V.D. // Phys. Rev. B. 2000. V. 62. P. R13298.

[17] Ciuti C., Schwendimann P., Deveaud B., Quattropani A. // Phys. Rev. B. 2001. V. 63. P. 041303 (R).

[18] Savvidis P.G., Ciuti C., Baumberg J.J., Whittaker D.M., Skolnik M.S., Roberts J.S. // Phys. Rev. B. 2001. V. 64. P. 075311.

[19] Savona V., Schwendimann P., Quattropani A. // Phys. Rev. B. 2005. V. 71. P. 125315.

[20] Kavokin A., Lagoudakis P.G., Malpuech G., Baumberg J.J. // Phys. Rev. B. 2003. V. 67. P. 195321.

[21] Saba M., Ciuti C., Bloch J., Tierry-Mieg V., Adre R., Dang L.S., Kundermann S., Mura A., Bongiovanni C., Staehli J.E., Deveaud B. // Nature. 2001. V. 414. P. 731.

[22] Huang R., Tassone F., Yamamoto Y. // Phys. Rev. B. 2000. V. 61. P. R 7854.

[23] Baas A., Karr J.-Ph., Romanelli M., Bramati A., Giacobino E. // Phys. Rev. B. 2004. V. 70. P. 161307 (R).

[24] Baas A., Karr J.-Ph., Eleuch H., Giacobino E. // Phys. Rev. A. 2004. V. 69. P. 023819.

[25] Krizhanovski D.N., Gavrilov S.S., Love A.P.D., Sanvitto D., Gippius N.A., Tikhodeev S.G., Kulakovskii V.D., Whittaker D.M., Skolnick M.S., Roberts J.S. // Phys. Rev. B. 2008. V. 77. P. 115336.

[26] Lagoudakis P.G., Savvidis P.G., Baumberg J.J., Whittaker D.M., Eastham P.R., Skolnick M.S., Roberts J.S. // Phys. Rev. B. 2002. V. 65. P. 161310 (R).

[27] Tartakovskii A.I., Krizhanovskii D.N., Kurysh D.A., Kulakovskii V.D., Skolnick M.S., Roberts J.S. // Phys. Rev. B. 2002. V. 65. P. 081308 (R).

[28] Dominici L., Petrov M., Matuszewski M., Ballarini D., De Giorgi M., Colas E., Cancellieri E., Fernandez B.S., Bramati A., Gigli G., Kavokin A., Laussy F., Sanvitto D. // Nat. Commun. 2015. V. 6. P. 8993. 
[29] Cristofolini P., Christmann G., Tsintzos S.I., Deligeorgis G., Konstantinidis G., Hatzopoulos Z., Savvidis P.G., Baumberg J.J. // Science. 2012. V. 336. P. 704.

[30] Хаджи П.И., Васильева О.Ф., Белоусов И.В. // ЖЭТФ. 2018. T. 153. B. 2. C. 179.

[31] Rosenberg I., Mazuz-Harpaz Y., Rapaport R., West K., Pfeiffer L. // Phys. Rev. B. 2016. V. 93. P. 195151.

[32] Tsintzos S.I., Tzimis A., Stavrinidis G., Trifonov A., Hatzopoulos Z., Baumberg J.J., Ohadi H., Savvidis P.G. // Phys. Rev. Lett. 2018. V. 121. P. 037401.

[33] Gippius N.A., Shelykh I.A., Solnyshkov D.D., Gavrilov S.S., Rubo Y.G., Kavokin A.V., Tikhodeev S.G., Malpuech G. // Phys. Rev. Lett. 2007. V. 98. P. 236401.

[34] Paraiso T.K., Wouters M., Leger Y., Morier-Genoud F., Deveaud-Pledran B. // Nat. Mater. 2010. V. 9. P. 655.

[35] Cerna R., Leger Y., Paraiso T.K., Wouters M., MorierGenoud F., Portalla-Oberli M.T., Deveaud B. // Nat. Commun. 2013. V. 4. P. 2008

[36] Liew T.C.H., Kovokin A.V., Shelykh I.A. // Phys. Rev. Lett. 2008. V. 101. P. 016402.

[37] Espinosa-Ortega T., Liew T.C.H. // Phys. Rev. B. 2013. V. 87. P. 195305.

[38] Amo A., Liew T.C.H., Adrados C., Houdre R., Giacobino E., Kavokin A.V., Bramati A. // Nat. Photon. 2010. V. 4. P. 361.

[39] Chestnov I.Yu., Khudaiberganov T.A., Alodjants A.P., Kavokin A.V. // Phys. Rev. B. 2018. V. 98. P. 115302.

[40] Ballarini D., Chestnov I., Caputo D., Giorgi M.D., Dominici L., West K., Pfeiffer L.N., Gigli G., Kavokin A., Sanvitto D. // Phys. Rev. Lett. 2019. V. 123. P. 047401.

[41] Yulin A.V., Kozin V.K., Nalitov A.V., Shelykh I.A. // arXiv: 1909.05226. 2019.

[42] Васильева О.Ф., Хаджи П.И. // Опт. и спектр. 2013. Т. 115. C. 922.

[43] Хаджи П.И., Васильева О.Ф. // Опт. и спектр. 2011. Т. 111. C. 831.

[44] Хаджи П.И., Васильева О.Ф. // ФТТ. 2011. Т. 53. С. 1216.

[45] Khadzhi P.I., Vasilieva O.F. // J. Nanophotonics. 2012. V. 6. P. 061805.

[46] Khadzhi P.I., Vasilieva O.F. // J. Nanoelectronics and Optoelectronics. 2014. V. 9. P. 295.

[47] Mc Konstrie C.J., Radic S., Raymer M.G. // Opt. Express. 2004. V. 12. P. 5037.

[48] Okawachi Y., Luke M.Yu.K., Carvalho D.O., Ramelow S., Farsi A., Lipson M., Gaeta A.L. // Opt. Lett. 2015. V. 40. P. 5267.

[49] Васильева О.Ф., Зинган А.П., Хаджи П.И. // Опт. и спектр. 2018. T. 125. C. 425

[50] Питаевский Л.П. // УФН. 1998. Т. 168. С. 641.

[51] Градштейн И.С., Рыжсик И.М. Таблицы интегралов, сумм, рядов и произведений. М.: ГИФМЛ, 1963.

[52] Корн Г., Корн Т. Справочник по математике для научных работников и инженеров. М.: Наука, 1971. 